\title{
Secretory expression of human ScFv against keratin in Pichia pastoris and its effects on cultured keratinocytes
}

\author{
Jianyong Fan · Zhu Shen · Gang Wang • \\ Huilan Yang $\cdot$ Yufeng Liu
}

Published online: 26 February 2009

(C) Springer-Verlag 2009

\section{Erratum to: Arch Dermatol Res}

\section{DOI 10.1007/s00403-008-0908-4}

The author would like to change their affiliations in an online published version of his article as given below:

\author{
Jianyong Fan · Gang Wang · Yufeng Liu ( $\bowtie)$ \\ Dermatology Center of Chinese PLA, XiJing Hospital, \\ Forth Military Medical University, \\ 710032 Xi'an, China \\ e-mail: fanjianyongfmmu@sina.com.cn \\ Jianyong Fan · Huilan Yang \\ Department of Dermatology, \\ The General Hospital of Guangzhou Military Command, \\ 510010 Guangzhou, China
}

\author{
Zhu Shen \\ Department of Dermatology, Southwest Hospital, \\ Third Military Medical University, \\ 400038 Chongqing, China
}

The online version of the original article can be found under doi:10.1007/s00403-008-0908-4.

J. Fan · G. Wang · Y. Liu ( $ه)$

Dermatology Center of Chinese PLA,

XiJing Hospital, Forth Military Medical University,

710032 Xi'an, China

e-mail: fanjianyongfmmu@ sina.com.cn

J. Fan $\cdot$ H. Yang

Department of Dermatology,

The General Hospital of Guangzhou Military Command,

510010 Guangzhou, China

Z. Shen

Department of Dermatology, Southwest Hospital,

Third Military Medical University, 400038 Chongqing, China 